\title{
Gating of Human Theta Oscillations by a Working Memory Task
}

\author{
Sridhar Raghavachari, ${ }^{1}$ Michael J. Kahana,, ${ }^{1,2}$ Daniel S. Rizzuto, ${ }^{1}$ Jeremy B. Caplan, ${ }^{1}$ Matthew P. Kirschen, ${ }^{1}$ \\ Blaise Bourgeois, ${ }^{2}$ Joseph R. Madsen, ${ }^{1,2}$ and John E. Lisman ${ }^{1}$ \\ 1 Volen Center for Complex Systems, Brandeis University, Waltham, Massachusetts 02454, and 2Department of Surgery, \\ Harvard Medical School and Children's Hospital, Boston, Massachusetts 02115
}

\begin{abstract}
Electrode grids on the cortical surface of epileptic patients provide a unique opportunity to observe brain activity with high temporal-spatial resolution and high signal-to-noise ratio during a cognitive task. Previous work showed that largeamplitude theta frequency oscillations occurred intermittently during a maze navigation task, but it was unclear whether theta related to the spatial or working memory components of the task. To determine whether theta occurs during a nonspatial task, we made recordings while subjects performed the Sternberg working memory task. Our results show event-related theta and reveal a new phenomenon, the cognitive "gating" of
\end{abstract}

Oscillations in the theta frequency band $(4-9 \mathrm{~Hz})$ have been extensively studied in rats (Vanderwolf, 1969; Bland, 1986; O'Keefe and Recce, 1993; Skaggs et al., 1996), where they are especially prominent during spatial exploration. These oscillations can be seen in the field potential and in the potentials recorded from individual pyramidal cells (Leung and Yim, 1986; Fox, 1989; Ylinen et al., 1995; Kamondi et al., 1998). An important observation that sheds light on the function of theta is that hippocampal place cells systematically change their phase of firing relative to theta as the rat moves through a place field (O'Keefe and Recce, 1993; Skaggs et al., 1996; Jensen and Lisman, 2000). This suggests that one function of theta is to provide a reference frame for a neural code in which different spatial information is represented at different phases of the theta cycle. It remains controversial whether theta oscillations in the rat are specialized for the organization of spatial information in the hippocampus or are more generally involved in other functions (O'Keefe and Burgess, 1999).

Given the importance of theta oscillations in the rat, it has been of interest to determine whether similar oscillations occur in humans. Theta band energy can be detected in humans by both MEG and EEG methods and is evident during working memory tasks (Gevins et al., 1997; Sarnthein et al., 1998; Klimesch, 1999; Tesche and Karhu, 2000). It has recently become possible to observe large-amplitude $(>100 \mu \mathrm{V})$ theta oscillations in humans

\footnotetext{
Received Nov. 13, 2000; revised Jan. 10, 2001; accepted Jan. 26, 2001.

This work was supported by National Science Foundation Grant IBN-9723466, National Institutes of Health Grant MH-55687, and the Alfred P. Sloan Foundation. We thank Larry Abbott, Xiao-Jing Wang, Marc Howard, and Adam Kepecs for helpful comments on a previous version of this manuscript. We acknowledge the enthusiastic cooperation of colleagues in the Children's Hospital Epilepsy Program, including Dr. Peter M. Black and Lewis Kull. Finally, we are most grateful to the patients and their families for their participation and support.

Correspondence should be addressed to John E. Lisman, Volen Center for Complex Systems, Brandeis University, 415 South Street, Waltham, MA 024549110. E-mail: lisman@brandeis.edu.

Copyright (C) 2001 Society for Neuroscience $\quad 0270-6474 / 01 / 213175-09 \$ 15.00 / 0$
}

a brain oscillation: at many cortical sites, the amplitude of theta oscillations increased dramatically at the start of the trial, continued through all phases of the trial, including the delay period, and decreased sharply at the end. Gating could be seen in individual trials and varying the duration of the trial systematically varied the period of gating. These results suggest that theta oscillations could have an important role in organizing multi-item working memory.

Key words: theta oscillations; working memory; Sternberg; intracranial EEG; brain waves; human by intracranial EEG (iEEG), a method that uses electrode arrays to record the EEG directly from the cortical surface (Kahana et al, 1999a, b; Caplan et al., 2000). These electrodes are implanted in epileptic patients to determine the location of seizure foci. The high signal-to-noise ratio of these recordings makes it possible to detect large-amplitude oscillations with a clear spectral peak in the theta frequency range and to study the dynamics of these oscillations during individual trials. This is not generally possible with the smaller MEG or EEG $(1-10 \mu \mathrm{V})$ signals recorded from the scalp. The iEEG study of Kahana et al. (1999b) showed that theta oscillations occurred in intermittent bouts during a maze navigation task and that the probability of their occurrence was related to task difficulty. However, it remains unclear whether theta was related to the memory or spatial components of the task.

To determine whether large-amplitude theta can occur in a task that lacks a spatial component, we have recorded from intracranial electrode arrays while subjects performed the Sternberg task, a classic test of nonspatial, multi-item, verbal working memory (Sternberg, 1966). We found that theta oscillations occur during this task and have investigated its properties. The Sternberg task is particularly well suited for examining the temporal properties of theta because each trial has a well defined period over which working memory must be maintained. Thus, it was possible to investigate the timing of changes in theta with respect to the period of working memory.

\section{MATERIALS AND METHODS}

\section{Subjects}

Our four subjects had normal range of personality and intelligence and were all able to perform the task within normal limits. Subject 1 (male, age 23), subject 2 (male, age 18), and subject 3 (female, age 22) had implanted electrode arrays, whereas subject 4 (male, age 19) had bilateral depth electrodes in the temporal lobe. The research protocol was approved by the institutional review board at Children's Hospital (Boston, MA), and informed consent was obtained from the subjects. 


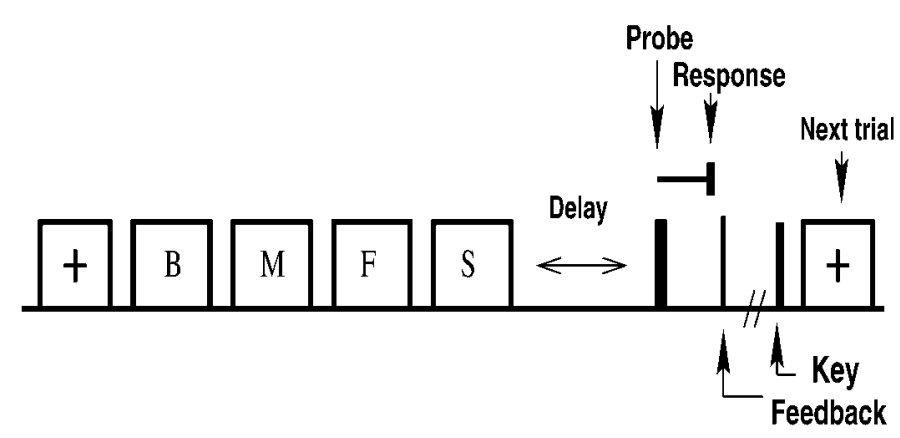

Figure 1. Schematic of the Sternberg task illustrating a four-item list using the "yes/no" procedure. A series of letters was presented after an orienting cue $(+)$. After a delay period, a probe item was shown. Subjects indicated whether the probe was on the list, and RT was measured. After the response, the probe was turned off, and subjects received feedback on their performance and initiated the next trial by a key press.

\section{Intracranial EEG recording}

iEEG signal was recorded from arrays (grids or strips) containing multiple platinum electrodes ( $3 \mathrm{~mm}$ diameter) with an interelectrode spacing of $1 \mathrm{~cm}$. Grids varied in size but covered several square centimeters of the cortical surface. The location of the electrodes was determined using coregistered postoperative computed tomograms and preoperative MRIs by an indirect stereotactic technique (Talairach and Tournoux, 1988). The iEEG signal was amplified, sampled at $200 \mathrm{~Hz}$ (Telefactor Corporation apparatus; band-pass filter: $0.5-100 \mathrm{~Hz}$ ) for subjects 1 and 2, and at $256 \mathrm{~Hz}$ (Biologic Corp. apparatus; bandpass filter, 0.3-70 Hz) for subjects 3 and 4 . Because of clock time discrepancies between the recording and experimental computers, our clock calibration was accurate to only $\pm 200 \mathrm{msec}$.

\section{Sternberg protocol}

Lists of 1-4 consonants were presented sequentially on a computer screen. Although items were presented visually, this form of the Sternberg task is nevertheless considered a verbal working memory task because the stimuli are meaningful linguistic units (Baddeley, 1986). To start each trial, a visual orienting cue was presented $1 \mathrm{sec}$ before the first list item (Fig. 1). Items were presented for $1.2 \mathrm{sec}$ each with a $200 \mathrm{msec}$ interval between items. The termination of the last item in the list was followed by a delay period of either $0.9 \mathrm{sec}$ (subjects 1 and 2) or $2 \mathrm{sec}$ (subjects 3 and 4), after which the probe was presented. The probe consisted of two letters for subjects 1 and 2 (forced choice variant), with one letter drawn from the presented list. The subject responded by pressing the left Control key if the first probe item was on the list and the right Control key if the second probe item was on the list. Subjects 3 and 4 were tested using the standard "yes"/"no" version of the Sternberg task, with a single probe item (Fig. 1a). The subjects responded by pressing the left Control key if the probe item was on the list and the right Control key otherwise. After each response, subjects received accuracy feedback (correct, incorrect) and latency feedback (very fast, fast, good response time, slow) via a screen message and then initiated the next trial by pressing a key. The subsequent trial began $1.6 \mathrm{sec}$ after this key press. The mean interval between the response for one trial and the start of the next trial was $\sim 2.5 \mathrm{sec}$. During each session, trials of each list length were randomly interleaved. We obtained 50, 96, 140, and 140 trials at each list length for subjects $1-4$, respectively. Only correct trials with RTs $<2.5$ sec were used for analysis. Because there was no significant difference in our results for correct "yes" and "no" trials, data were pooled across these trial types.

\section{Exclusion criteria}

Subjects were excluded from analysis if their behavioral performance was poor (mean response times $>2 \mathrm{sec}$ or had high error rates). Approximately half the subjects (four of a total of nine subjects) that were tested were able to perform the task satisfactorily. Sites that were located over known lesions (determined from clinical records) or were involved in seizure onsets (identified by examining the seizure records) were excluded as were sites that showed epileptiform spiking (interictal spikes or spike-and-waves) activity. A total of 73 such sites (of 320) were rejected.

\section{Data analysis}

Power spectra. Because the oscillatory nature of the iEEG data was of interest, data analysis was done in the frequency domain. The power spectrum is the Fourier transform of the autocorrelation function. A simple estimate of the power spectrum, the magnitude-squared Fourier transform of the data has poor "bias" (the power at nearby frequencies contribute to the power at any given frequency, distorting the estimate) and variance (the estimate of the spectrum does not converge to the true value even if the data length increases) properties (Thomson, 1982). Multitaper techniques (Thomson, 1982; Mitra and Pesaran, 1999) provide a formal method to obtain estimates of the spectrum with optimal bias and variance properties. Briefly, the data set is windowed (tapered) using a set of special windows (Slepian windows), which are maximally concentrated in a time duration, $T$, and a bandwidth in frequency, $W$ (Thomson, 1982). The time and frequency resolution of the windows thus fixes the number of windows, $K=2 T W-1$, that can be used. The windowed data is then transformed to the frequency domain by calculating the Discrete Fourier transform, resulting in $K$ estimates of the spectrum, $S_{k}(f)$. Averaging these estimates reduces the variance of the spectrum by $\sqrt{K}$. Our typical choices for $T$ and $W$ were $1 \mathrm{sec}$ and $2 \mathrm{~Hz}$, respectively. The averaged power spectra were obtained by averaging the single trial estimates.

Spectrograms. The spectral properties of stationary data sets do not change over time, i.e., the power spectrum of any stretch of data is statistically similar to any other stretch. If however, the spectrum varies over time, the data set is nonstationary. One method to quantify nonstationarity is to compute a time-varying spectrum, or spectrogram. Spectrograms were computed using the squared modulus of the complex demodulates [projection of the iEEG data onto different frequency bands using filters (1 sec duration, $4 \mathrm{~Hz}$ bandwidth) constructed from the Slepian windows (Mitra and Pesaran, 1999)]. Estimates from different Slepian windows were averaged together to obtain the spectrogram for each trial. The spectrograms for each trial were aligned with the onset of the first list item and averaged together. Only oscillatory activity with high signal-to-noise ratio will be apparent in averaged spectrograms (Tallon-Baudry et al., 1996).

Test for gating. Gating of theta was tested by comparing the energy in the average spectrogram during the trial to the energy in the $1 \mathrm{sec}$ before the orienting stimulus. Because the distribution of energies in the spectrogram is non-Gaussian, a nonparametric method (Mann-Whitney $U$ test; $p<0.05$ ) was used to compare the average energy in each $250 \mathrm{msec}$ epoch during the trial with the intertrial energy. Because the analysis windows were 1 -sec-long, adjacent $250 \mathrm{msec}$ bins are not independent. Multiple comparisons (for the number of electrodes, frequencies and bins) were corrected for by a Bonferroni correction.

Nonstationarity test. A second method to quantify nonstationarity is to expand the spectrogram, $S(f, t)$, along an orthogonal set of basis functions $A_{1}(t)$ such that:

$$
S(f, t)=\sum_{l=0}^{L-1} a_{l}(f) A_{l}(t),
$$

results in coefficients, $a_{1}(f)$ that are functions of the frequency alone, with $L$ denoting the number of terms retained in the expansion. Quadraticinverse theory (Thomson, 1990, 2001) can be used to pick an appropriate basis set, $A_{l}(t)$, such that the number of terms in the expansion, $L$ is fixed to be $4 T W$, where $T$ and $W$ are defined above, fixing the time and frequency resolution. Coefficients of higher order are identically zero. The coefficients, $a_{l}(f)$, of for the quadratic-inverse basis then take on special meaning. The 0 th order coefficient, $a_{0}(f)$ is approximately $S(f)$, or the time-averaged spectrum. The first order coefficient, $a_{1}(f)$, is the time-derivative of the spectrum and so on. Thus, features such as sharp or gradual changes in power, frequency drifts etc. can be readily identified in a noisy background.

For a constant amplitude signal of a single frequency, the expansion coefficients vanish for all orders $\geq 1$. For a stationary process, the ratio:

$$
\Xi(f)=\left(\sum_{l=0}^{L-1} \frac{a_{l}(f)}{S(f)}\right)^{2},
$$

where $S(f)$ is the mean power, is $\chi_{\mathrm{L}-1}^{2}$-distributed. If the signal is systematically nonstationary at a given frequency $f$ across several trials, the ratio will be significantly different from the expected value $L-1$ for 

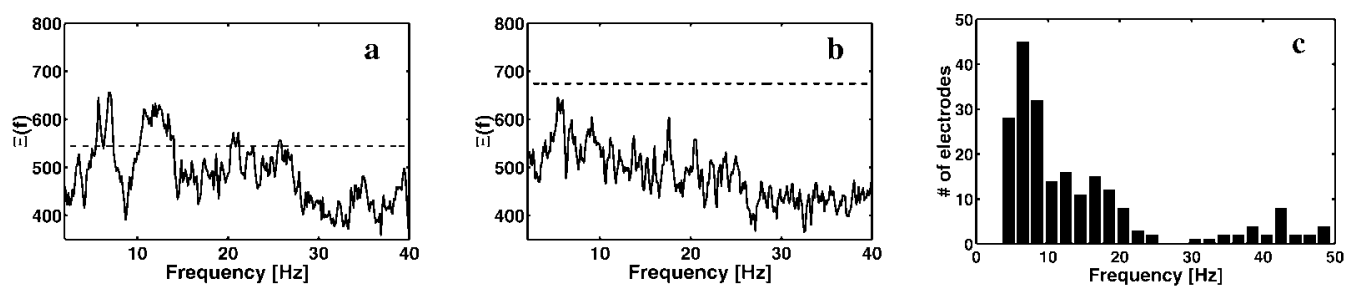

Figure 2. iEEG data show significant task-related nonstationarity, predominantly in the theta frequency band. To be considered significant, the nonstationarity index must be higher than the horizontal line, which denotes the $99.999 \%$ point of the $\chi^{2}$ distribution with the appropriate degrees of freedom (see Materials and Methods). Nonstationarity index $\Xi(f)$ shown for two representative electrodes. $a$, Subject 1 , Talairach coordinates (left-right, anterior-posterior, inferior-superior) are $+44,+11,+38$. This electrode exhibits significant nonstationarity in the theta and beta bands, as well as some peaks in the $20-30 \mathrm{~Hz}$ range. $b$, Subject 3 , Talairach coordinates are $-42,+8,+42$. This shows an electrode that has no significant peaks. In both cases, the average power spectrum had peaks in the theta frequency range. For a majority of frequencies, the measure is distributed between the 5 and $95 \%$ confidence intervals with most values around the theoretical mean value, indicating that the method is appropriate. $c$, Summary plot of the number of electrodes which showed significant nonstationarity as a function of frequency. A given electrode could show nonstationarity at several different frequencies.

a $\chi_{\mathrm{L}-1}^{2}$ process. This results in a single number representing the amount of nonstationarity at each frequency. Consider a stretch of iEEG data around a trial of the Sternberg task. If the spectral characteristics of the iEEG change because of the onset and offset of the task, or within the task itself, the nonstationary index $\Xi(f)$, will be significantly different from that expected by chance. The degrees of freedom, $L$ for a single trial is equal to the highest order term retained in the expansion, $L_{\max }$. For multiple trials, this becomes $L_{\max } \times N_{\text {trials }}$. We considered the signal to be nonstationary at a given frequency $f$ if the ratio exceeded a percentile threshold (typically $99.999 \%$ or $p<0.00001$ ) of the $\chi_{\mathrm{L}-1}^{2}$ distribution at that frequency. It is appropriate in our case to use a high value of significance given the large number of frequencies (256) and sites that were tested. This test allows a classification of iEEG data as stationary or non-stationary at a given frequency.

Test for continuity. To assess the continuity of theta within a trial, the baseline level of theta was first established by calculating the spectrum for a $1 \mathrm{sec}$ interval after the response for each trial, and the individual estimates of the spectrum were log-transformed. The jack-knife variance (Mitra and Pesaran, 1999) was computed from individual estimates by leaving out one trial at a time and averaging over the remaining estimates. The resulting jack-knife statistic is $t$-distributed, and a threshold value was chosen as the $99.999 \%$ point of this distribution. The continuity of theta within a trial was now assessed as the fraction of trials (from all trials of all list lengths) at which the narrow-band power dipped below the threshold for any interval of $>0.25 \mathrm{sec}$. The jack-knife statistic is used because it is a robust measure that does not make any assumptions about the underlying distribution of the data.

\section{RESULTS}

Figure 1 illustrates the structure of each trial of the Sternberg task. We visually presented lists of one to four consonants. After a delay period, the subjects' task was to indicate as rapidly as possible whether a probe item was on the list. We quantified the speed of the response by measuring the response time (RT). This task was administered to three subjects who had intracranial electrode arrays and one with depth electrodes. Each of these subjects performed the Sternberg task with very high accuracy; for subjects 1-4, accuracy was $86,98,97$, and $96 \%$, respectively. RT increased significantly with list length (LL) for all subjects $(p<0.005)$. This increase, approximated by the equation $R T=$ $a \times L L+b$ msec, had coefficients $(a, b)=(89,817),(95,1008)$, $(40,463)$, and $(37,353)$ for subjects $1-4$, respectively. The differences in RTs between the first two and last two subjects was most likely a consequence of differences in the design of the trial structure (see Materials and Methods).

To examine the oscillations occurred during the Sternberg task, it was desirable to have an unbiased algorithm to detect consistent task-related changes in several frequency ranges. Because the data set obtained was extensive $(\sim 200$ trials/subject; total of 247 sites), examination of the entire data set by eye was impossible.
We adapted a test developed by Thomson (2001) to detect taskrelated changes in different frequency bands (see Materials and Methods). The nonstationarity index, $\Xi(f)$, identifies electrodes in which the task produces transient or maintained changes in spectral power at any frequency $f$. No assumptions are made about the timing, duration, or sign of the changes. The only requirement for detection is that the changes be consistent across trials.

This nonstationarity test was applied to all sites that were not rejected for epileptic artifacts (see Materials and Methods). Figure $2, a$ and $b$, shows $\Xi(f)$ for two representative electrode locations. The broken line in the two panels represents the $99.999 \%$ confidence level for the statistic. Figure $2 a$ shows an electrode for which $\Xi(f)$ exceeds this level in the theta, beta, and gamma frequency bands, indicating consistent task-related changes at these frequencies. Figure $2 b$ shows an electrode in which $\Xi(f)$ did not exceed the required significance level at any frequency, suggesting little or no task-related activity at this site. We detected a total of 74 electrodes (of a total of 247 across all subjects) for which $\Xi(f)$ exceeded the $99.999 \%$ significance level at one or more frequencies. The electrode locations of these sites were widely dispersed over the cortex ( 24 in the temporal lobe, 18 in the occipital lobe, 18 in the parietal/motor/premotor areas, and 14 in the frontal lobe). Figure $2 c$ shows a plot of the number of nonstationary electrodes at different frequencies. The majority of these (60) had significant nonstationarity in the theta frequency range $(4-9 \mathrm{~Hz})$, most prominently between 6 and $8 \mathrm{~Hz}$. We therefore conclude that there are widespread task-related changes in theta during a memory task that lacks a spatial component. Task-related changes were also observed in the gamma frequency range, but these will be analyzed elsewhere.

To determine how theta changed during the task, we computed trial-averaged spectrograms for the nonstationary sites. An examination of these spectrograms revealed an interesting pattern of task-related activity at some sites: theta power increased at the beginning of the trial, was elevated through item presentation and the delay period, and decreased after the response. Figure $3 a$ shows the averaged spectrograms from sites that display such a pattern in each of the four subjects. The average spectrograms for these sites show a clear peak in the $4-9 \mathrm{~Hz}$ range, the theta frequency band. Although peak frequencies and overall levels of activity varied across subjects, the general pattern at these gated sites is similar. Because the pseudocolor plots of the spectrograms emphasize certain transitions in power while making others less 
a
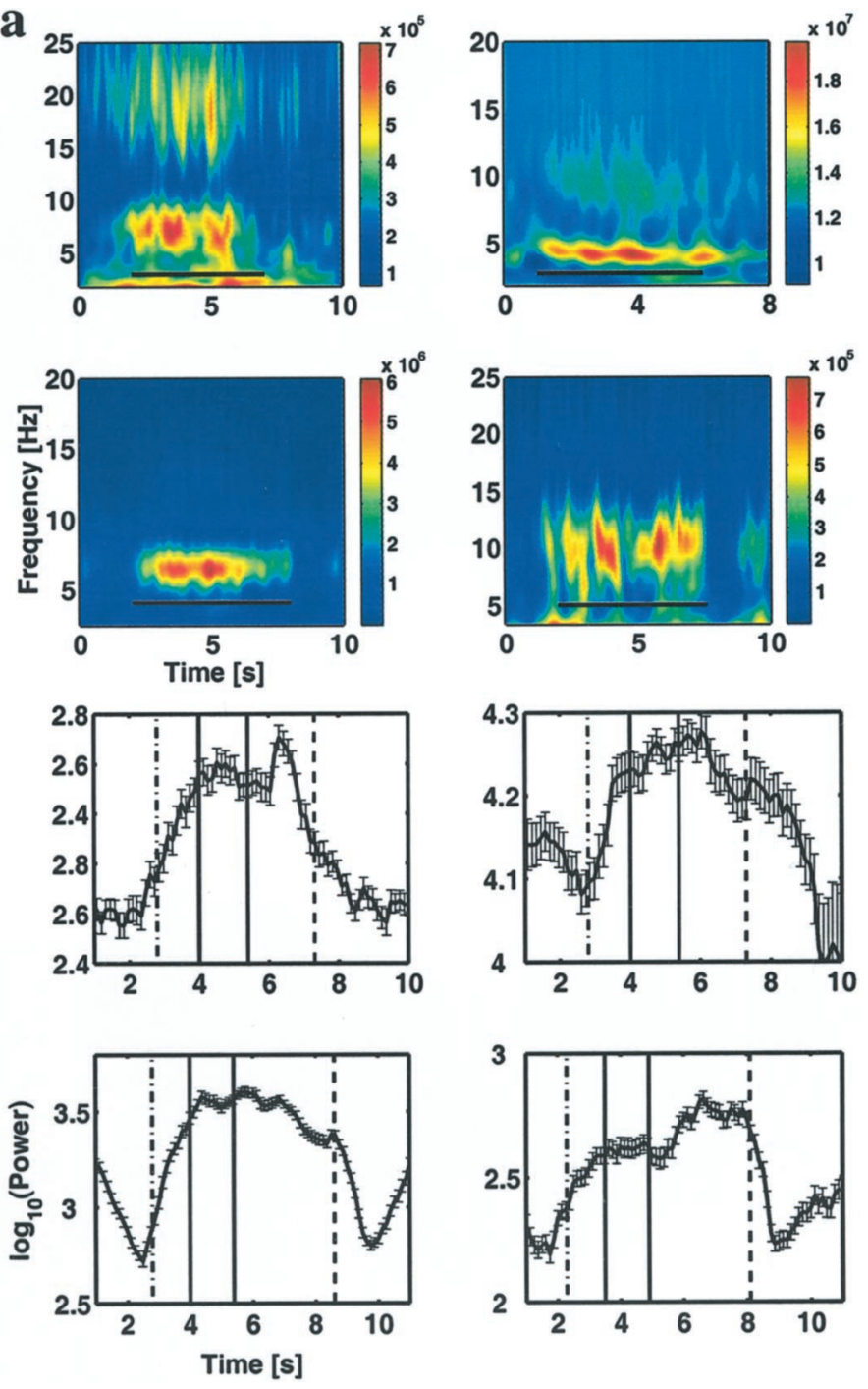

Figure 3. Theta is gated during the Sternberg task. $a$, Time-frequency energy averaged over all two-item lists shows sustained theta activity. An example is shown from each of the four subjects. The data illustrated were obtained from a right frontal site in subject 1 (Talairach coordinates are $+44,+11,+38)$ (top left); a left temporal site in subject 2 (Talairach coordinates are $-42,-9,-10)$ (top right); a right frontal site (Talairach coordinates are $-52,-34,+38$ ) in subject 3 (bottom left); and a depth electrode in left temporal lobe (Talairach coordinates are $-25,-72,+6$ ) in subject 4 (bottom right). Black bars in the spectrograms denote the trial duration from the orienting cue to the mean response time for two item lists. Because of limitations in our synchronization techniques and methods of time-frequency analysis, the determination of the onset and offset of theta has a precision of $\pm 200 \mathrm{msec}$. The color scale represents power in square microvolts. In subject 1 , we also observed similar gating centered around $18 \mathrm{~Hz}$. However, because this finding was not duplicated across subjects, we did not analyze it further. $b$, Evolution of average theta power in time for the above four electrodes for a bandwidth of $4 \mathrm{~Hz}$ around the peak frequency. The dot-dashed vertical line marks the orienting cue, the two solid lines denote the list items, and the dashed line denotes the probe. Theta power is elevated throughout the trial, with fluctuations within the trial. The error bars denote the $95 \%$ confidence intervals.

visible, it is important to graphically plot changes in theta power as a function of time. Figure $3 b$ shows the evolution of narrowband power at the same four sites averaged over all trials with two-item lists (4 Hz bandwidth around the peak frequency in the spectrogram). In all cases, theta power was elevated during the trial relative to the intertrial period. Note that the falling phase before trial onset in the bottom left panel occurs because the average intertrial interval for this subject was unusually brief (2 $\mathrm{sec}$ ): because this interval was of the same order as the data window for the spectrogram (see Materials and Methods), the falling phase can be attributed to the offset of the previous trial. Note also that the shifts in theta power during the trial were relatively small. The most prominent feature at these sites is the gating "on" of theta at the onset of the trial and the gating "off" at the end of the trial. The average changes in power were high, increasing by a factor of 2 (top panels, subjects 1 and 2) or 8 (bottom panels, subjects 3 and 4). One question that remains unclear is whether theta is activated by the cue initiating the trial or the presentation of the first memory item. Technical limitations (see Materials and Methods) prevent us from determining the onset of gating with a precision better than $\pm 200 \mathrm{msec}$. It is therefore unclear whether theta turns on with the orienting cue in anticipation of the need for engaging working memory or whether it turns on with the presentation of the first memory item. Experiments with longer delays between the orienting cue and the first list item would be useful in clarifying this issue.

It was desirable to develop a statistical test to determine whether this gating was statistically significant and whether it could be seen at a large number of sites. We therefore adopted a test for gating: that the average theta power (across trials) in every overlapping $250 \mathrm{msec}$ epoch within the trials exceed the power during the intertrial period at the $95 \%$ confidence level. Thirty sites (of the 74 classified as nonstationary) met this criterion ( $p<0.01$, by a Bonferroni corrected, two-tailed, MannWhitney $U$ test). One or more gated sites was detected in each of our subjects. It should be emphasized that sites that pass the gating test necessarily have an increase in theta power during the "pure" memory period, i.e., the interval after the offset of the last list-item and the onset of the probe $(0.9 \mathrm{sec}$ in the forced-choice variant and $2 \mathrm{sec}$ otherwise) compared with the baseline power immediately after the response and before the onset of the subsequent trial $(t$ test; $p<0.01)$. This observation indicates that theta is engaged during the pure working memory period without possible confounds of item presentation. The remainder of the electrodes that showed significant nonstationarity in the theta range typically had elevated theta power during a fraction of the trial duration, and thus were not classified as gated sites. We will not discuss these sites further.

To determine whether gating was dependent on the duration of the task, we examined responses to trials of different list lengths (and consequently trial duration). Figure 4 illustrates the change in gating with list length. Two examples are shown, one from a recording site on the surface of the left parietal lobe and one from a depth electrode in the left temporal lobe. In both cases, the duration of sustained theta closely followed the duration of the trial. It can also be seen that the maximum of the average theta power at these sites did not vary significantly with list length. Similarly, the frequency of theta did not change as additional items were presented (Fig. $3 a$ ). The pattern of gating at other sites was similar. We conclude that theta oscillations of relatively stereotyped frequency and power were gated by each trial of the task and that the period of gating coincided well with the duration of the trial.

Although Figures 3 and 4 indicate that the average theta power is continuous at gated sites, the possibility remains that theta is not continuous during individual trials. In fact, this seemed likely, because previous iEEG recordings (Kahana et al., 1999b) showed 

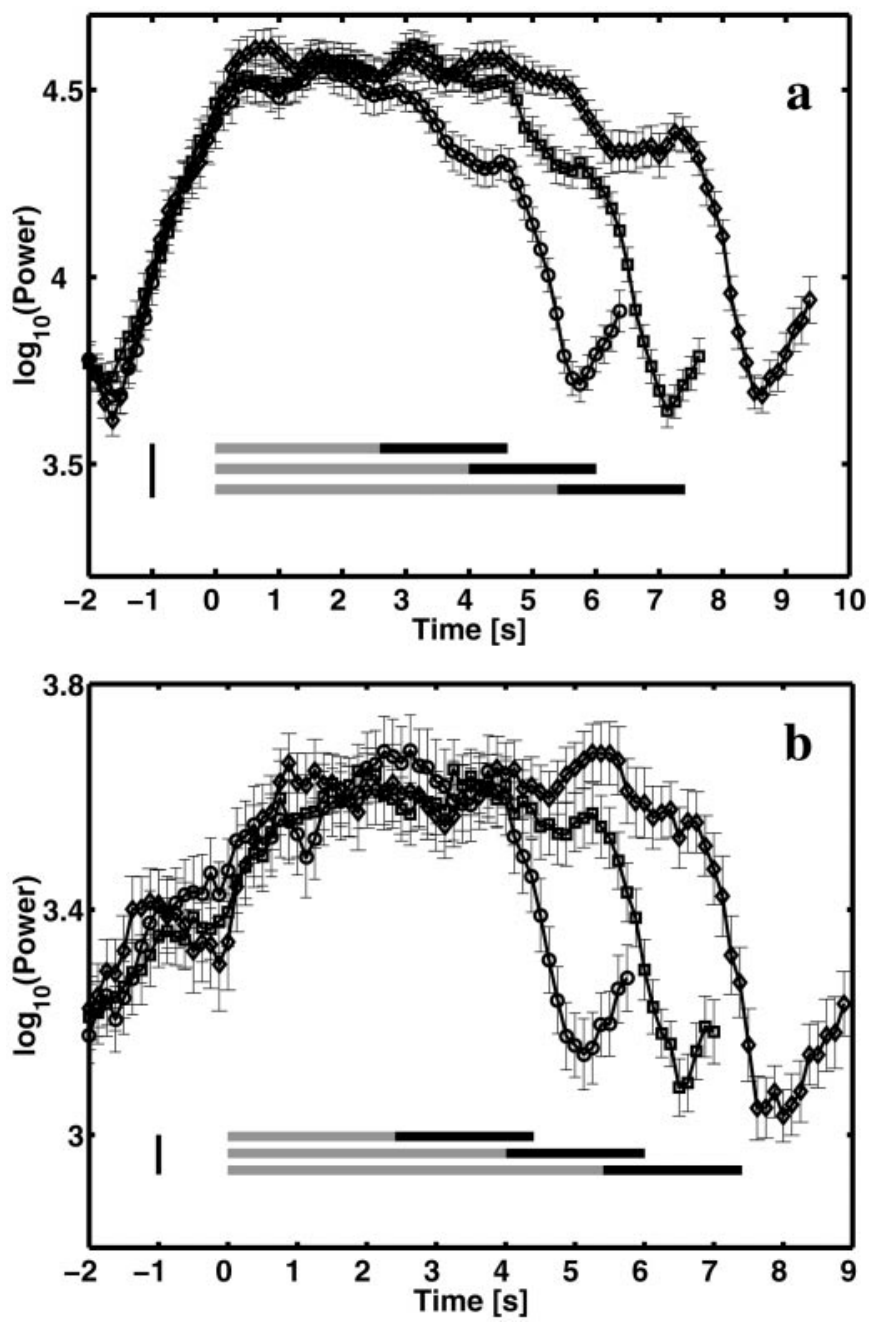

Figure 4. Gating varies systematically with list length. Averaged theta power $(5-9 \mathrm{~Hz})$ as a function of time shows that theta is elevated for the entire duration of the trial. The three different traces are averages over trials with two-, three- and four-item lists (circles, squares, and diamonds, respectively). Gray bars mark the presentation of the list items, and the black bars mark the delay interval until the presentation of the probe for the two-, three-, and four-item lists. The large tick at $-1 \mathrm{sec}$ marks the onset of the orienting cue. $a$, Recording from a subdural electrode in the parietal cortex (subject 3 , Talairach coordinates are $-52,-34,+38$ ). $b$, Recording from a depth electrode in left temporal lobe (subject 4, Talairach coordinates are $-25,-72,+6$ ). The rise subsequent to the end of the trial is attributable to the onset of the next trial.

that theta occurs intermittently during a spatial maze navigation task. As seen in Fig. 5a, which shows an unfiltered trace, theta appears to be continuously elevated during a trial of the Sternberg task. Indeed, theta oscillations were similarly gated during each of five consecutive trials (Fig. 5b). Also shown (Fig. $5 c$ ) is the time evolution of the narrow band power ( $2 \mathrm{~Hz}$ bandwidth) at the peak frequency $(7 \mathrm{~Hz})$ over the course of these successive trials. This plot shows that theta power during the task was greater than the level during the intertrial periods for a large fraction of each trial. In a more rigorous analysis of the ten gated sites with the largest amplitude theta (central region in subject 3; depth electrodes in subject 4), we calculated the fraction of trials for which there was a return to baseline theta power (see Materials and Methods) for any interval $>0.25 \mathrm{sec}$ during individual trials. The fraction of such trials was very low (ranging from 0.05 to 0.1 over

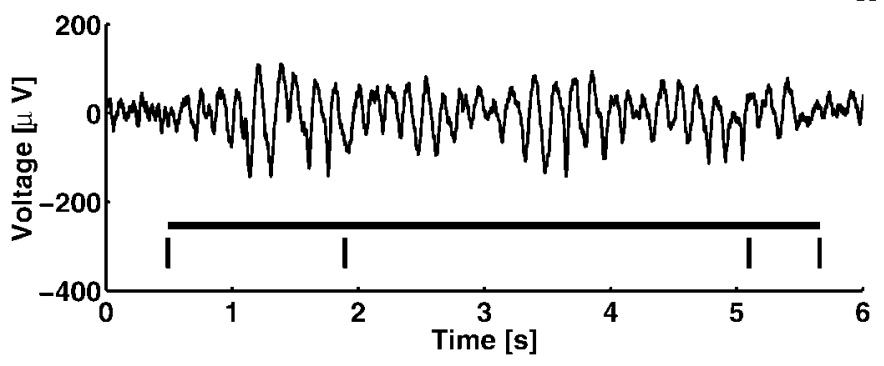

b
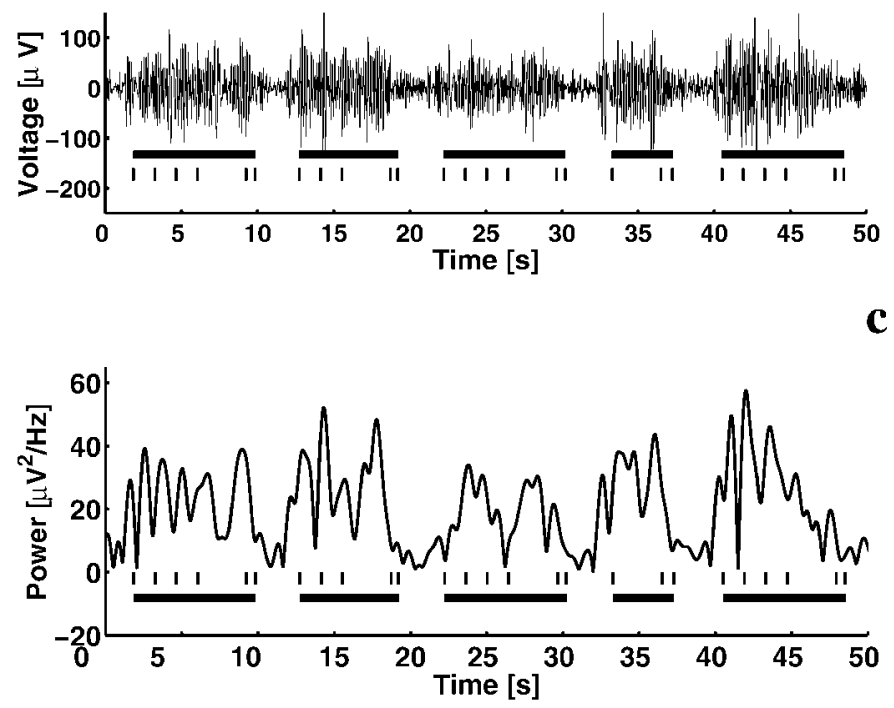

Figure 5. Gating of theta oscillations is evident in single trials of the raw iEEG signal. $a$, Sample raw iEEG trace recorded from an electrode in the parietal cortex (subject 3, Talairach coordinates are $-52,-34,+38$ ) during a two-item list. The black bar below the trace marks the task duration, whereas the ticks denote the presentation of the list items, probe, and response, respectively. $b$, A $50 \mathrm{sec}$ iEEG trace with five consecutive trials from the same electrode shows clear enhancement in theta activity for the duration of each trial. Bars and tick marks are as above. $c$, Narrow-band power $(7 \pm 1 \mathrm{~Hz})$ for the $50 \mathrm{sec}$ trace above shows clear enhancement during trials relative to intertrial intervals.

all trials for all three list lengths). We conclude that there are many sites at which theta is continuous or nearly so during individual trials.

Several interleaved controls indicate that the signals at thetagated sites were not directly related to sensory stimulation or to the execution of a motor response. Between successive trials, subjects were given visual feedback regarding their performance on the previous trial. This information was presented on the same monitor as the list items. However, as illustrated in Figure 6, this sensory stimulus did not evoke theta activity at gated sites. A second issue concerns the possibility that theta might occur in preparation for motor responses. However, Figure 6 shows that theta did not occur in anticipation of the motor response (key press) by which subjects initiated the next trial. More quantitatively, we compared the theta power in the $1 \mathrm{sec}$ before the response at the end of the trial to the theta power $1 \mathrm{sec}$ before the key press ( 75 trials of all list lengths in each subject with a $1 \mathrm{sec}$ interval between the response and the key press). The theta power before the key press was significantly smaller $(p<0.01 ; t$ test) than before the response. We conclude that theta activation 
Figure 6. Theta activity not caused by sensory stimulus or motor responses. Averaged spectrogram for a site showing gated theta activity (subject 1, Talairach coordinates are $+44,+11,+38)$. The trials were aligned to the response. The bars marked Feedback and Key denote the sensory stimulus (visual feedback after the response) and the mean time of the motor response to initiate the subsequent trial, respectively. One hundred trials of all list lengths with a mean delay of $1 \mathrm{sec}$ between the response and the key press (to initiate the next trial) were used to compute the spectrogram. Theta activity $(\sim 6$ $\mathrm{Hz}$ peak frequency) has a sharp offset after the response and stays off until the beginning of the next trial (1.6 sec after the key press). The small increase in theta activity after the key press is caused by averaging trials of different mean intervals between the feedback and key press. The color scale represents power in square microvolts per Hertz.

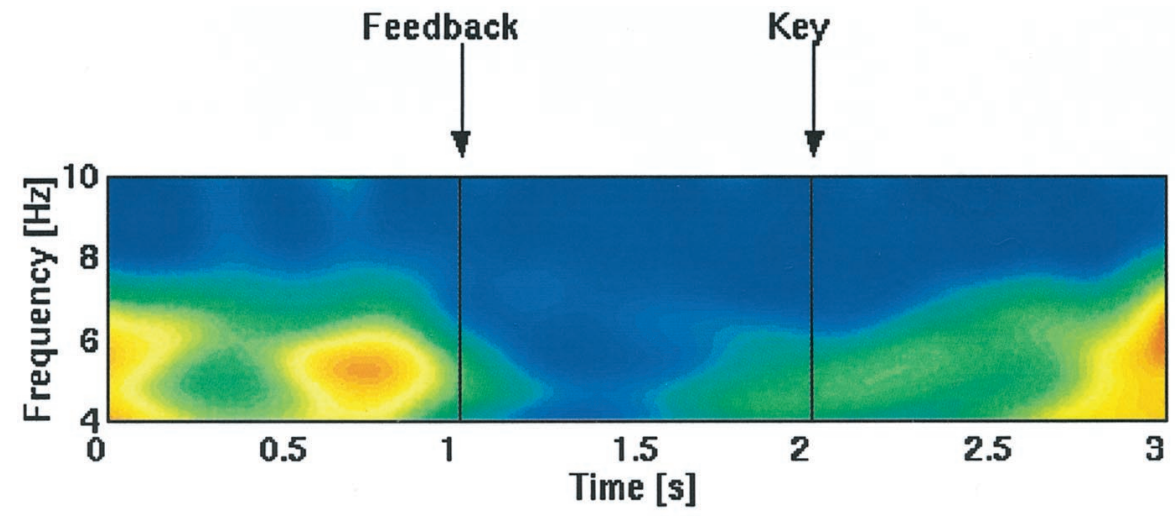

$\begin{array}{lllll} & 1 & 100 & 1250\end{array}$
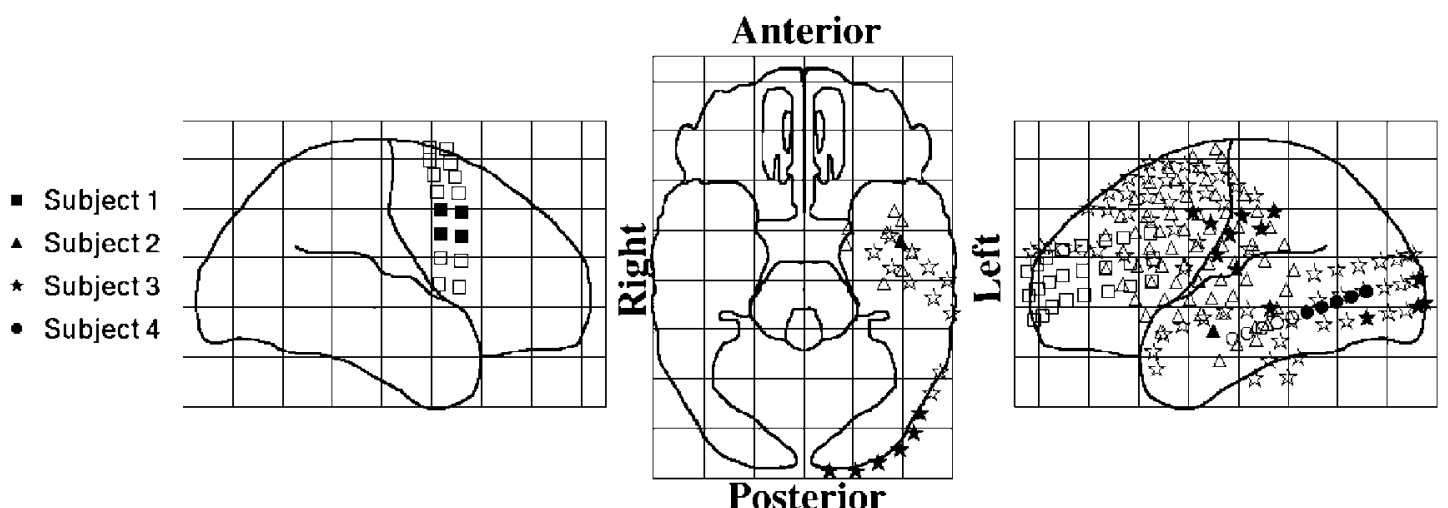

Figure 7. Views of standardized brain showing electrode locations of gated sites. Filled symbols indicate electrodes in which theta was gated "on" by the task. Open symbols indicate electrodes that did not meet our significance threshold. Different symbols indicate different subjects.

cannot be simply explained as a sensory or motor preparatory response.

Although we have found a large number of gated sites (30), it is difficult to make any strong conclusions about the distribution of these sites on the cortical surface because of the sparse sampling. It is important to understand that the electrode arrays were placed in candidate seizure loci. While providing details within the coverage area, the electrodes only covered a small fraction of any one lobe. Thus, our failure to detect activity in a given lobe of a subject does not imply that it was not present in that lobe. Despite these limitations, it is important to document the location of gated sites (Fig. 7). These appear to be distributed widely over the cortex in frontal, temporal, parietal, and occipital lobes. Furthermore, in two patients in which theta was detected with large arrays, we found that many of the gated sites were clustered near each other. However, closely spaced sites did not necessarily show similar activity. Figure 8 shows average spectrograms (left panels) and average power spectra (right panels) from each of three nearby electrodes (1-2 cm separation; subject 3$)$. Sites with gated theta activity (middle row) sometimes occurred near other sites with no clear task-related theta activity (top row). Furthermore, in this subject (subject 3), there were sites (bottom row in Fig. 8) where theta activity was gated off by the task (i.e., the theta power was suppressed throughout a trial and rebounded after the response). Because such "off" gating was only detected in one subject, we describe it here only because it provides further evidence that large-amplitude theta can be very different at closely spaced sites.

\section{DISCUSSION}

Theta in rats has been most reliably elicited by movement, and it has therefore been suspected that theta may have a special role in spatial processing. It was thus of considerable interest that the first observation of large-amplitude theta in humans was during a spatial task (Kahana et al., 1999b). However, this task also had a memory component, leaving the possibility that theta might also occur in memory tasks that lack a spatial component. We therefore obtained iEEG data from subjects performing a verbal working memory task to test whether this nonspatial, working memory task also elicited large theta frequency oscillations. Using an objective test for nonstationarity, we showed that the Sternberg task evokes clear task-related changes in the iEEG in the theta frequency band (Fig. 2) at some cortical and subcortical sites. The power spectra showed a theta peak, the amplitude of which increased markedly during the task compared with baseline levels. Our finding that theta occurred during a task that lacked a spatial component strongly argues against the view that human theta is uniquely specialized for spatial computations. This con- 

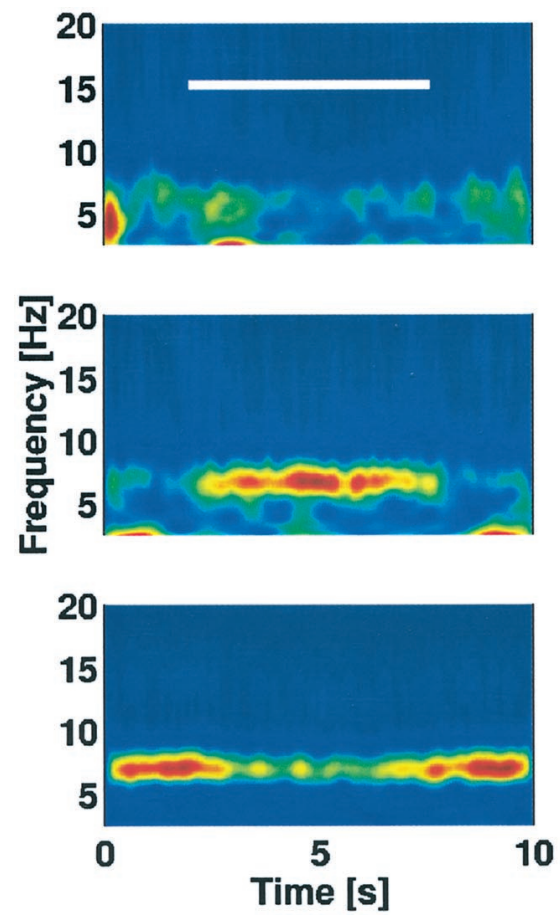
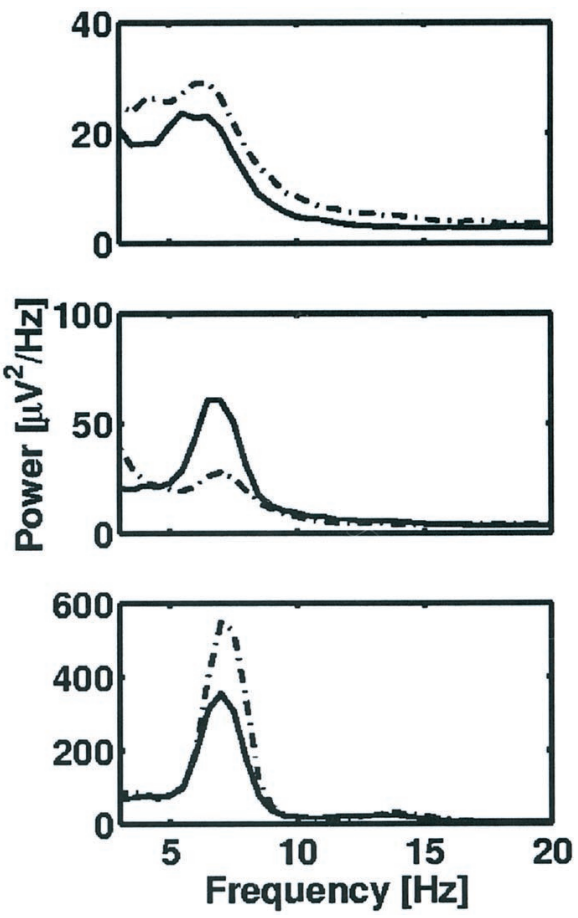

Figure 8. Nearby regions (spacing $\sim 1-2 \mathrm{~cm}$ ) can have dramatically different patterns of theta activity. Left panels show averaged spectrograms. Right panels show averaged power spectra for the corresponding electrodes (solid lines denote in-task power, dotdashed lines denote out-of-task). The white bar in the top left panel marks the duration of the trial from the onset of the orienting cue until the presentation of the response. All three electrodes show theta activity evidenced by the peaks in the power spectra. The top panel shows an electrode with continuous, theta activity that is weakly modulated by the task. Significant task-gated theta increase in activity is evident for the electrode in the middle panel. The bottom panels show suppression of theta activity during the trial. (Talairach coordinates from top to bottom are $-50,-14,+41 ;-54,-16,+29 ;-56$, $-24,+23$.) clusion is consistent with several observations in rat (Macrides et al., 1982; Givens and Olton 1990, 1995; Givens, 1996) and humans (Gevins et al., 1997; Sarnthein et al., 1998; Klimesch, 1999; Tesche and Karhu, 2000), indicating that theta can occur in nonspatial contexts.

A second major finding of this study is that we detected a large number of sites (30 in four subjects) in which the amplitude of theta oscillations increased at the beginning of the trial, stayed elevated through the entire trial, and decreased at the end (Fig. 3). We term this phenomenon "gating." When the duration of the trial was changed, theta gating changed accordingly (Fig. 4). Although previous EEG studies indicated that theta could occur during working memory tasks (Gevins et al., 1997; Klimesch, 1999), the timing of the involvement of theta was not investigated because the structure of the tasks was not suited for the study of timing issues. In contrast, the working memory component of the Sternberg task has a well defined onset and offset, which allowed us to detect a direct correlation of theta with task duration.

Although average theta power (across trials) was gated at these sites, we confirmed that individual trials also exhibited this gating. The high signal-to-noise ratio of the iEEG allowed us to determine that theta is continuous, relative to a baseline, at electrodes with high-amplitude, gated theta (Fig. 5). This is in contrast to the intermittent nature of theta observed in a spatial task (Kahana et al., 1999b). However, it is possible that if we knew when the memory demands occurred in the latter task, it would be continuous during those periods. The continuous nature of theta has important implications for models of working memory that are based on oscillatory activity (see below).

Several findings indicate that activity observed at theta gated sites cannot be a simple consequence of the sensory and motor components of the task. In interleaved controls (Fig. 6), we found that a sensory input or a motor response that was unrelated to working memory did not evoke theta at gated sites. These results, along with the tight temporal linkage of theta gating to the onset and offset of the period of working memory, suggest that theta oscillations may play an important role in human verbal working memory.

\section{Spatial organization of theta}

Our findings indicate that gated theta, although common, is not uniformly present (Fig. 7). The locations of gated sites were widely dispersed over the cortex. Although iEEG is uniquely well suited to give a fine grained, high temporal resolution view of theta, it is not well suited to establishing the regional localization of theta, because the grid placement is sparse and determined solely by clinical considerations. It is nevertheless tempting to try to relate the limited data available to brain regions implicated in working memory by fMRI methods (Ungerleider, 1995; Goldman-Rakic, 1995; Smith and Jonides, 1998). However, we caution against this for several reasons. First, recent work on theta in rats indicates that periods of high and low theta have nearly the same overall rates of firing (Csicsvari et al., 1999). Thus, changes in theta amplitude may not be detected by hemodynamic methods. A second point concerns the special methods that are used in fMRI studies to isolate the brain regions that are specifically involved in an aspect of brain function by subtracting the activation evoked by a simpler task that controls for sensory and other processes. In our case, all areas engaged by the task, including purely sensory areas, might be expected to show taskrelated oscillations. Indeed, the activation we observe in the occipital cortex might be related to sensory processes rather than memory processes. Our finding that widely distributed brain regions generate theta during a working memory task is consistent with EEG studies also show increased theta synchronization between posterior and frontal regions (Sarnthein et al., 1998) during a working memory task.

\section{Oscillatory basis of working memory}

Electrophysiological studies of working memory indicate that persistent firing of cells underlies working memory (GoldmanRakic, 1995). Our results suggest that this firing may have an oscillatory character. Oscillatory single unit activity has not generally been reported in the delayed response tasks in monkeys (but see Nakamura et al., 1992), but it is not clear how to relate animal electrophysiological studies on single-item nonverbal 
working memory (Goldman-Rakic, 1995) to the multi-item verbal working memory that we have studied in humans. It is possible that verbal working memory is more complex than the simpler forms used in animal studies (Baddeley, 1986) and that this may explain why oscillatory activity has not generally been seen in single units during simple working memory tasks in monkeys.

\section{Relevance to models}

Memory performance in the Sternberg task has been extensively studied, and the behavioral results strongly constrain possible models. Jensen and Lisman (1998) have proposed several variants of oscillatory models that account for the details of response time distributions in the Sternberg task. Their models were inspired by the observation (in rat) that different spatial information is encoded at different phases of the theta cycle (see introductory remarks). They propose that similar phase coding may be important in multi-item working memory (Lisman and Idiart, 1995; Jensen and Lisman, 1998) with different memory items active at different phases of the theta cycle. The continuous nature of theta during individual trials of the Sternberg task (Fig. 6) provides support for such models. In one of the variants of the JensenLisman model, the frequency of theta oscillations was assumed to decrease as a function of the number of items being held in working memory. This model would seem to be ruled out by our finding that theta frequency does not vary significantly with memory load (Fig. 3a) at gated sites. A second model was based on the assumption that the phase of theta is reset by the arrival of the probe, an assumption that is supported by recent MEG results (Tesche and Karhu, 2000).

Although we have focused here on the possible role of theta in multi-item working memory, there are other possible roles, none of which are mutually exclusive. One possibility, for which studies of long-term potentiation provide some evidence (Pavlides et al., 1988; Huerta and Lisman, 1993), is that theta is used to rapidly encode information directly into long-term memory by synaptic modification. Another possible function of theta is to synchronize different regions of the cortex that participate in the task (Sarnthein et al., 1998). Analysis of the synchronization of theta at different sites during the Sternberg task is currently underway.

\section{Concerns about the validity of data derived from epileptic patients}

Intracranial recordings from epileptic patients are increasingly being used to study brain activity during cognitive tasks (Fried et al., 1997; Fernandez et al., 1999; Kahana et al., 1999b; Caplan et al., 2000; Kreiman et al., 2000). In such studies the possible contribution of epilepsy to the conclusions needs to be addressed. A number of observations suggest that the presence of theta activity during the Sternberg task is not a result of seizure activity. First, the precision of the gating (Fig. 3) and the high degree of spatial localization (Fig. 7) are exactly the opposite of what would be expected from an uncontrolled process like epilepsy. Second, because the location of the seizure origin is not known before the electrode implantation, iEEG from many regions is sampled to identify the focus. Thus, many of the sampled sites are far from the clinically determined epileptogenic foci. We observed task-related theta in each of our subjects at sites that were distant from the seizure foci, sometimes even in different hemispheres (in subjects 1 and 4). Third, direct examination of seizure activity in these subjects showed it to have a much higher amplitude ( $>1 \mathrm{mV}$ peak-to-peak) with different spectral structure than even the largest amplitude theta signal during the task (200 $\mu \mathrm{V}$ peak-to-peak). Fourth, recent work using MEG has detected theta activity in normal subjects during the Sternberg task (Tesche and Karhu, 2000). Finally, the patients in this study had behavioral performance similar to normals on the Sternberg task. Thus, the task-related theta activity does not appear to be a consequence of the pathology of the subjects.

\section{Concluding remarks}

Elucidation of the properties of theta in rats progressed rapidly because spatial exploration is such a reliable task for eliciting rat theta. The present findings indicate that a working memory task is as good at eliciting theta in humans as spatial exploration is in rats. The highly reliable way in which human theta can be elicited by the Sternberg task and the ability to precisely control the cognitive demands of the task make this an ideal experimental system for the further study of the role of theta in memory and cognition.

\section{REFERENCES}

Baddeley AD (1986) Working memory. Oxford, UK: Clarendon.

Bland BH (1986) The physiology and pharmacology of hippocampal formation theta rhythms. Prog Neurobiol 26:1-54

Caplan JB, Kahana MJ, Sekuler R, Kirschen M, Madsen JR (2000) Task dependence of human theta: the case for multiple cognitive functions, in press.

Csicsvari J, Hirase H, Czurko A, Mamiya A, Buzsáki G (1999) Fast network oscillations in the hippocampal CA1 region of the behaving rat. J Neurosci 19:RC20:1-4.

Fernandez G, Effern A, Grunwald T, Pezer N, Lehnertz K, Dumpelmann M, Van Roost D, Elger CE (1999) Real-time tracking of memory formation in the human rhinal cortex and hippocampus. Science 285:1582-1585.

Fox SE (1989) Membrane potential and impedance changes in hippocampal theta rhythm. Exp Brain Res 77:283-294.

Fried I, MacDonald KA, Wilson CL (1997) Single neuron activity in the human hippocampus and amygdala during recognition of faces and objects. Neuron 18:753-765.

Gevins A, Smith ME, McEvoy D, Yu L (1997) High-resolution EEG mapping of cortical activation related to working memory: effects of task difficulty, type of processing, and practice. Cereb Cortex $7: 374-385$

Givens B (1996) Stimulus-evoked resetting of the dentate theta rhythm: relation to working memory. NeuroReport 8:159-163.

Givens BS, Olton DS (1990) Cholinergic and GABAergic modulation of medial septal area: effect on working memory. Behav Neurosci 104:849-855.

Givens B, Olton DS (1995) Bidirectional modulation of scopolamineinduced working memory impairments by muscarinic activation of the medial septal area. Neurobiol Learn Mem 63:269-276.

Goldman-Rakic P (1995) Cellular basis of working memory. Neuron 14:477-485.

Huerta PT, Lisman JE (1993) Heightened synaptic plasticity of hippocampal CA1 neurons during a cholinergically induced rhythmic state. Nature 364:723-725.

Jensen O, Lisman JE (1998) An oscillatory short-term memory buffer model can account for data on the Sternberg task. J Neurosci $18: 10688-10699$

Jensen O, Lisman JE (2000) Position reconstruction from an ensemble of hippocampal place cells: contribution of theta phase coding. J Neurophysiol 83:2602-2609.

Kahana MJ, Caplan JB, Sekuler R, Madsen JR (1999a) Using intracranial recordings to study theta. Response to O'Keefe J and Burgess $N$ (1999). Trends Cognit Sci 3:406-407.

Kahana MJ, Sekuler R, Caplan JB, Kirschen M, Madsen JR (1999b) Human theta oscillations exhibit task dependence during virtual maze navigation. Nature 399:781-784.

Kamondi A, Acsady L, Wang X-J, Buzsaki G (1998) Theta oscillations in somata and dendrites of hippocampal pyramidal cells in vivo: activitydependent phase-precession of action potentials. Hippocampus $8: 244-261$.

Klimesch W (1999) EEG alpha and theta oscillations reflect cognitive and memory performance: a review and analysis. Brain Res Brain Res Rev 29:169-195.

Kreiman G, Koch C, Fried I (2000) Category-specific visual responses of single neurons in the human medial temporal lobe. Nat Neurosci 3:946-953

Leung LS, Yim CY (1986) Intracellular records of theta rhythm in hippocampal CA1 cells of the rat. Brain Res 367:323-327. 
Lisman JE, Idiart MA (1995) Storage of $7 \pm 2$ short-term memories in oscillatory subcycles. Science 267:1512-1515.

Macrides F, Eichenbaum HB, Forbes WB (1982) Temporal relationship between sniffing and the limbic $\theta$ rhythm during odor discrimination reversal learning. J Neurosci 2:1705-1717.

Mitra PP, Pesaran B (1999) Analysis of dynamic brain imaging data. Biophys J 76:691-708.

Nakamura K, Mikami A, Kubota K (1992) Oscillatory neuronal activity related to visual short-term memory in monkey temporal pole. NeuroReport 3:117-120.

O'Keefe J, Burgess N (1999) Theta activity, virtual navigation and the human hippocampus. Trends Cognit Sci 3:403-406.

O'Keefe J, Recce ML (1993) Phase relationship between hippocampal place units and the EEG theta rhythm. Hippocampus 3:317-330.

Pavlides C, Greenstein YJ, Grudman M, Winson J (1988) Long-term potentiation in the dentate gyrus is induced preferentially on the positive phase of theta-rhythm. Brain Res 439:383-387.

Sarnthein J, Petsche H, Rappelsberger P, Shaw GL, von Stein A (1998) Synchronization between prefrontal and posterior association cortex during human working memory. Proc Natl Acad Sci USA 95:7092-7096.

Skaggs WE, McNaughton BL, Wilson MA, Barnes C (1996) Theta phase precession in hippocampal neuronal populations and the compression of temporal sequences. Hippocampus 6:149-172.

Smith E, Jonides J (1998) Neuroimaging analyses of human working memory. Proc Natl Acad Sci USA 95:12061-12068.
Sternberg S (1966) High-speed scanning in human memory. Science 153:652-654.

Talairach J, Tournoux P (1988) Co-planar stereotaxic atlas of the human brain. Stuttgart, Germany: Verlag.

Tallon-Baudry C, Bertrand O, Delpuech C, Pernier J (1996) Stimulus specificity of phase-locked and non-phase-locked $40 \mathrm{hz}$ visual responses in human. J Neurosci 16:4240-4249.

Tesche C, Karhu J (2000) Theta oscillations index human hippocampal activation during a working memory task. Proc Natl Acad Sci USA 97:919-924.

Thomson DJ (1982) Spectrum estimation and harmonic analysis. Proc IEEE 70:1055-1096.

Thomson DJ (1990) Quadratic-inverse spectrum estimates: applications to plaeoclimatology. Philos Trans R Soc Lond [A] 332:539-597.

Thomson DJ (2001) Multitaper analysis of nonstationary and nonlinear time series data. In: Nonlinear and nonstationary signal processing (Fitzgerald WJ, Smith RL, Walden AT, and Young PC, eds) Cambridge, UK: Cambridge UP, in press.

Ungerleider L (1995) Functional brain imaging studies of cortical mechanisms for memory. Science 270:769-775.

Vanderwolf CH (1969) Hippocampal electrical activity and voluntary movement of the rat. Electroencephalogr Clin Neurophysiol 26:407-418.

Ylinen A, Soltesz I, Bragin A, Pentonnen M, Sik A, Buzsaki G (1995) Intracellular correlates of hippocampal theta rhythm in identified pyramidal cells, granule cells, and basket cells. Hippocampus 5:78-90. 